\title{
Subject Index Vol. 31, 1995
}

Abrupt discontinuation, neuroleptic medication 135

Acute schizophrenia 135

Age 47

Aggression 120

Alertness level 47

Alpidem 81

Amineptine 130

Amitriptyline 130

Analgesic efficacy, B vitamins 156

Antidepressants 1,146

Anxiety disorders 125

-, premorbid 113

Anxious depression 130

Autism 16, 53

Autonomic nervous system 24

Background factors 113 Blood platelets 64,120

Caffeine 195,200,202

withdrawal 195,200,202

Cerebral metabolic rate 173

Chaos mathematics 166

Cognition 98

Cognitive tasks 210 Colourblindness 58 Comorbidity 125 Complement 53

- $\quad$ C4B protein 53

Correlation exponent 166

Cortical EEG 89

Cortisol 135

CSF monoamine metabolites 76

Dementia 173 Deoxyglucose 173 Depression 10, 64 Depressive disorder 68 Descriptive data analysis 31 Dexamethasone suppression test 10 Diazepam 31,81

Diclofenac 156 Distance computation 31 Dopamine 135 Driving performance 81 Dysthymic disorder 68

EEG 210

correlation dimension 47

global dimensional complexity 47

mapping 31 Electroconvulsive shock 1

therapy 182 
Epinephrine 135

Event-related desynchronization 16 Evoked brain potentials 156 Experimental pain 156

Factor IX 58

Fast Fourier transformation power maps 89

Fluoxetine 64

Frontal cortex 1

Generalized anxiety disorder 6 Glucose-6-phosphate dehydrogenase 58 Glycosaminoglycan polysulfate 76

Headache 125 Heart rate 204 Homo vanillic acid 135 Hostile behavior 120 5-Hydroxyindoleacetic acid 6

Imipramine 1, 68

- $\quad$ binding 120

Intracutaneous stimulus 156

Isoflurane narcotherapy 182

Lorazepam 81 Lormetazepam 204 Lundby|Study 113 Lymphocytes 53

Manic depression 58

Minaprine 68

Minnesota Multiphasic Personality Inventory 24

Mood 195

- disorders 125

Naltrexone 16 Non-parametric statistics 31 Norepinephrine 135

Ondansetron 81

[3/4]paroxetine binding sites 1 Performance 98 Personality 113 Pharmaco-EEG 31 Plasma concentrations 156 Platelet 3/4-imipramine binding 64 MAO-B activity 76

$\alpha$-Power 16

Primary degenerative dementia 76 Principal component analysis 31 Prolactin 135

217

Psychometric tests $98,146 \quad$ Sodium valproate 210

Psychomotor performance 195,200,202 Spontaneous EEG 156

Psychopathology 24 Suriclone 81

Psychophysiology 98

$\mathrm{T}$ cells 53

QRS complexes 204 Temporal lobe 173

Quantitative electroencephalometry 98

Unrestrained behavior, rat 89 Response predictors 64

Vanillylmandelic acid 6,135

Serine $10 \quad$ Verbal memory 173

Serotonin 64 Visual evoked potential 89

- $\quad$ uptake $1,120 \quad X$ linkage 58

Skin potential response 24

Sleep $204 \quad$ Zopiclone 204

- $\quad$ EEG 166

218 
